\title{
Study of Life Satisfaction and Happiness among Male Patients of Diabetes: Insulin Vs Non Insulin
}

\author{
Dr. Ushakiran Agrawal \\ Professor, Department of Psychology, Govt. D.B. Girls P.G. College ,Pt Ravishankar Shukla University \\ Raipur Chhattisgarh, India. ushakiran2308@gmail.com
}

\section{Doi:10.5901/mjss.2015.v6n5s1p494}

\begin{abstract}
Present study focuses on study of life satisfaction and happiness of diabetes patients (male) those who were on insulin $(N=20)$ and those who were not on insulin $(N=20)$, chosen on random basis, from a private hospital at Bhopal M.P. A measure of life satisfaction by Dr Pramod Kumar and Jayshree Dhyani(1988) was administered on them as well as happiness was measured by happiness scale by Dr J C Ajwani and Amba Sethi(2001). Results on putting to -t test indicated that there was a significant difference between happiness of insulin Vs non insulin patients, those who were on insulin were seen less happy than those who were not on insulin, similarly on five dimensions of life satisfaction, there was a significant difference between insulin and non insulin group of male diabetics.
\end{abstract}

Keywords: Happiness, Life Satisfaction, Mental Job, Social, Marital , Family

\section{Introduction}

Present paper studies the life satisfaction and happiness level of the 20 (male from the private hospital of Bhopal M.P.)) looking at the way the family diabetics behave it gave me an insight to study them, especially those on insulin around me an observation of their behavior showed that they are touchy and get panicked easily in spite of their good physical condition, they appear less happy and less contended from their life, after knowing their happiness and life satisfaction levels on different dimensions e.g. mental, job, social, marital and family necessary counseling can be done accordingly measurement of happiness levels and if it is low in a particular group an effort can be done to get it enhanced.

\section{Introduction}

\subsection{Definition of Life Satisfaction}

Life satisfaction means a concept that reflects condition of good life, it is explained in a different manner in context of economy ,sociology, psychology, medicine, and health care, quality and content and measures of good life varies between and within disciplines( Farquhar, 1995), more than 1000 measures in various fields of quality of life has been given by (Hughes \& Hwang, 1996), more than hundreds of definition has been given by (Cummins, 1997).

Despite of the lack of a broadly accepted definition most of the definitions of quality of life encompass a a status which is multidimensional functional and subjective (Muldoon, Barger, Flory, \& Manuck, 1998). Physical well-being, functional ability, emotional well-being, and social well-being come under multidimensional functional status, while subjectivity is related to how the individual's perceives his or her quality of life (Muldoon et al., 1998).

The person perception of how his or her life and how they see that where it will be in the future is considered as life satisfaction. Life satisfaction is a measure of measure of well-being and furthermore a cognitive, global judgment. It meant with a positive attitude of one's life as a whole. Life satisfaction can be measured in relation to a person's economic status, education, experiences, and the people's residence as well as many other.

Life satisfaction shows those experiences of a person that have influenced a person in a positive way. These experiences have the caliber to encourage people to pursue and to reach their desired goals. The two emotions that can influence how people perceive about their lives are Hope and optimism, these two include cognitive processes related to reaching of goals and the perception of these goals. Previous studies showed that positive views and life satisfaction were completely arbitrated by the concept of self-esteem, and the difference in the methods of perceiving ideas and events by people. Self-esteem plays a role in influencing life satisfaction has been proved by many studies. Outlook 
towards life and mood can also influence one's own perception of their life satisfaction. It has been suggested that overall life satisfaction is derived from within an individual based on the personal values of the individual and what he or she feels is important, it may be family, love, and money or other material items; it varies from one person to person . Prior researches have shown that predominantly males were found materialistic, Lower life satisfaction level was reported in materialistic persons as compared to non-materialistic persons.

Similarly those who value money more than helping other people showed less life satisfaction; this is because the money they have can buy them the assets they deem valuable. Materialistic people constantly need more and more belongings hence are less satisfied with their life were less satisfied with life, after they obtain these belongings they lose value and they again need more belongings and a vicious cycle sets. Materialistic individuals in lack of money to possess these belongings, become more dissatisfied towards their life. This is referred as a hedonic treadmill. On the opposite side , if a person does not hold the acquisition of wealth on high priority, his or her personal financial state will not make a difference on how happy he or she is with life overall. The persons who are inclined to traditions and religion reported to have a higher level of life satisfaction. similarly those doing daily prayers and are church goers, reported higher levels of life satisfaction same was found true with people who valued creativity and who valued respect for and from othersqualities that not related to material goods. Life satisfaction level was reported to be higher in those persons who received social support, from friends, family, or church. The people who valued interpersonal relationships were found to be more satisfied as compared to those who valued material possessions. Life satisfaction is assessed by what feelings and attitudes one has about his life at a particular point in time ranging from negative to positive, one of three major indicators of well being - life satisfaction ,positive effect and negative effect (Diener ,1984), Diener, Suh, Lucas, \& Smith (1999) include will to change one's life; satisfaction with past; future; other's views of one's life." (Beutell), Suh et. al. reported that the correlation between life satisfaction and the prevalence of positive affect is higher in individualistic cultures. In collectivistic cultures adhering to norms are also considered important for life satisfaction

Life satisfaction is not mere attainment of goals or the feeling good about oneself, five variables control it, Neugarten, Havighurst, and Tobin's (1961.

The five factors while determine the life satisfaction are

- Zest vs. apathy showed by enthusiasm of response to life in general. it is not related to any specific type of activity was not related to any specific type of activity. A person who feels better in sitting at home and reading was found to score higher on this scale as compared to an energetic person. Physical energy as well as intellectual energy and other highly involved pursuits contributed to a high score.

- Resolution and fortitude-This scale measures active acceptance of personal responsibility of the respondent, in spite of condoning what has happened to them.. Erikson's integrity is similar and relates to the meaningfulness of life and the lack of fear of death.

- Congruence between desired and achieved goals- This scale measures the relative difference between what is desired as goal and what is achieved and satisfaction or dissatisfaction with life of a person caused due to this rating .

- Self-concept - indicates a person's present emotional, physical, and intellectual features. . Persons who are concerned with their appearance and do not judge them old judge themselves to be wise and competent are likely to rate themselves higher on this factor. Successful living in the past contributes indirectly to this component.

- Mood tone. The last factor, mood tone, is associated with optimism and happiness and other positive affective responses. Low scores were seen in case of pessimism depression, sadness loneliness and irritability. Measurement of life satisfaction is more complex than just measuring happiness. A life state filled with happiness is an important contributor."

Life Satisfaction is "an operational definition of 'successful aging'" (Neugarten at al., 1961).

\subsection{Happiness}

"Happiness is a mental or emotional state of well-being characterized by positive or pleasant emotions ranging from contentment to intense joy. A number of biological, psychological, religious, and philosophical approaches have striven to define happiness and to recognize its sources. Various research groups, including positive psychology, are employing the scientific methods to research questions about what "happiness" is, and how it might be attained." http://en.wikipedia.org/wiki/Happiness

"Dimensions of Happiness 
1) pleasure and gratification, 2) embodiment of strengths and virtues and 3) meaning and purpose. Each kind of happiness is linked to positive emotion it is progression from the first type of happiness of pleasure/gratification to strengths/virtues and finally meaning/purpose." (Seligman 2002, p.61). http://www.pursuit-of-happiness.org/history-ofhappiness/martin-seligman-positive-psychology/

\subsection{Previous Study; Review of Literature}

Takahashi, Araki. et.al (2010) studied community participation associated with life satisfaction in elderly people with diabetes, a questionnaires covering physical, psychological, and social aspects was administered by interview to 56 elderly outpatients with diabetes mellitus. Life Satisfaction Index K (LSIK) was used to assess Life satisfaction.

The support Emotional, instrumental and social given by the families living along with the participants or living separately from the participants was studied. The presence of diabetic neuropathy and pain in the lower back or knee joints were found associated with low Life satisfaction index K scores, while Community participation, social curiosity, relationships with other people, and instrumental support from families living together with the participants positively correlated with high Life satisfaction Index K scores. The Life Satisfaction Index K scores of the leaders of the diabetes patient group were higher than the scores of those who only participated in the diabetes patient group. Community participation, instrumental support from families living along with the participants, and the absence of neuropathy were independently associated with high LSIK scores. Results indicated that Community participation is an important factor associated with life satisfaction in elderly people with diabetes mellitus.

Shahin, kalawa et . al (2013), studied quality of life satisfaction of diabetic foot patients, 77 from Egypt and 100 from Sudan, were the participants the study was carried out in Egypt in diabetic outpatient clinic in Port general hospital which it is the first specialized diabetic governmental center and in Sudan in Jabir Abu Alaiz diabetic specialized center which it is also the first specialized governmental diabetic center. One modified tool for data collection was used divided into 4 main parts related to socio-demographic characteristics, duration of diabetes mellitus, feeling, quality of life, satisfaction respectively.

Results indicated that the present study revealed significance difference in many items related to quality of life \& diabetic foot patients satisfaction between Egyptian \& Sudanese patients, Sudanese diabetic foot patients had low quality of life and satisfaction relate to their disease.

In a study by Humboldt, Leal, Santos \& Niculescu (2013), on Aging with Diabetes: Sense of Coherence and Satisfaction with Life in European Older Adults with Type 2 Diabetes, the objective of the study was to find out significant differences among three groups of older adults having diverse diabetes diagnosis, i.e., with only type 2 diabetes, with diabetes and other chronic diseases and remaining patients without any chronic disease, (SWL) the satisfaction with life and $(\mathrm{SOC})$ sense of coherence scores were gathered, and they also measured the relation of SWL with SOC in these groups, differences were found significant regarding the SWL(satisfaction with life) and SOC ( sense of coherence) in these three groups, only diabetes' group had lowest SOC and SWL scores, this group also showed an association between SOC and SWL scores. In the 'without diabetes' and 'with diabetes and other' groups no correlation was found between SOC and SWL scores. Results suggest that chronic diseases e.g. diabetes, as well as the presence of comorbidities, differently influence both SWL and SOC. This study gives an idea about the importance of SWL and SOC in the elderly, especially in the presence of diabetes, and focuses on the relevance of healthy aging.

Rubin and Peyrot (1999) reviewed the already available literature on self perception of quality of life among diabetic adults, physical and social functioning, perceived mental and physical wellbeing were taken as quality of life, it has been found that better glycemic control is related with better quality of life, no matter how intensive treatment is given , any kind of complication in diabetes had a detrimental effect on quality of life, similarly when patient's health status is improved and their perceived ability to control their disease was increased it showed improved quality of life in them.

\section{Proposed Methodology}

\subsection{Nature of the Problem}

(i) To study of Life Satisfaction among different dimensions in the male patients of Diabetes ( Insulin Vs non insulin)

(ii) To study the Happiness level among male patients of Diabetes; Insulin Vs Non Insulin. 


\subsection{Hypotheses}

(i) The male patients of diabetes on insulin will show more happiness than diabetic patients (male) who will be in non insulin category.

(ii) Diabetic patients (male) of insulin category will show significant difference on the different dimensions of life satisfaction scale namely- mental, family, job, social and marital as compared to the diabetic patients (male) with non insulin.

\subsection{Sample}

Consists of 20 - 20 male diabetic patients of insulin and non insulin category from Bhopal the patients were selected randomly those who were waiting for their turn to meet the physician ,

\subsection{Tools}

Pramod Kumar and Jayshree Dhyani's. Life satisfaction Scale having 54 items, having 5 dimensions-Menta, Family, Job, Social, and Marital was used.for measuring life satisfaction, happiness was measured by using a scale by $\mathrm{Dr}$ J.C. Ajwani and Amba Sethi (2001).

\subsection{Statistical Treatment}

Mean differences will be computed with the help of using t- test, and differences will be observed among five dimensions of life satisfaction among male diabetic patients of insulin and non insulin category.

\section{Procedure}

Data will be collected in small groups after giving instructions and making rapport with them.

\section{Result and Discussion}

Data was analyzed by means of computing t test to see the significant difference among diabetic male of insulin and non insulin category in happiness and among different dimensions of life satisfaction.

(1) As hypothesized The male patients of diabetes on insulin will show more happiness than diabetic patients (male) who will be in non insulin category .On the contrary the male patients of diabetes on insulin showed less happiness than those diabetic patients (male) who were in non insulin category .the mean of happiness in male patients of insulin category was found 97.95 lower, as compared to male diabetic patients of non insulin category having a mean of 100.55 , t was computed and was found 91.50 significant at higher level thus confirming the hypothesis. (Table 1).

Table 1: T-test

Differences between Diabetic Male (Insulin and Non Insulin) category in Happiness.

\begin{tabular}{|l|c|c|c|c|}
\hline Happiness & Df & Mean & T-Value & Sig. \\
\hline Insulin / Non- Insulin & 39 & 80.98 & 91.50 & $.01^{\star *}$ \\
\hline
\end{tabular}

Level of Sig. . $.01^{\star *}, .05^{\star}$

(2) As hypothesized that diabetic male patients( insulin non insulin category ) showed significant difference in the five dimensions of the life satisfaction i.e mental ,job , social ,marital and family dimensions, as the mean showed life satisfaction is higher in mental (30.1) social (25.5) and family dimensions (16.6) among male diabetic patients of insulin category while it was found higher in job (23.8) and marital dimension (27.65) in non insulin category of male diabetic patients.(Table 2). 
Table 2: Means of Life Satisfaction on Different Dimensions

\begin{tabular}{|l|c|c|c|c|c|}
\hline Category & Mental & Job & Social & Marital & Family \\
\hline Insulin & 30.1 & 19 & 25.5 & 27 & 16.6 \\
\hline Noninsulin & 26.15 & 23.8 & 24.45 & 27.65 & 15.85 \\
\hline
\end{tabular}

(3) On the mental dimension of life satisfaction (Table 3) t value 35.5 was found to be significant at .01 level showing that male diabetics with insulin and non insulin category differ in this dimension, however the mean of men diabetic with insulin is found to be higher i.e. 30.1 than non insulin category i.e. 26.15 showing that diabetic men with insulin have more mental - life satisfaction.

Table 3: T Test

Differences between Diabetic Male (Insulin and Non Insulin Category) Life Satisfaction in Mental Dimension

\begin{tabular}{|l|c|c|c|c|c|}
\hline Life Satisfaction & Df & Mean & SD & t-value & Sig. \\
\hline Mental & 39 & 29.61 & 5.20 & 35.05 & $.01^{\text {** }}$ \\
\hline
\end{tabular}

Level of Sig. $.01^{\star \star}, .05^{*}$

(3) On job dimension, the amount of life satisfaction is (Table 4) the t value 15.57 was also significant at 01 level showing difference in amount of life satisfaction (on job dimension) among diabetic males with insulin and non insulin category on this dimension the mean was found to be higher for diabetic men non insulin category (23.8) as compared to diabetic men insulin category (19) showing that diabetic men, non insulin category have more life satisfaction on job dimension.

Table 4: T test

Differences between Diabetic Male ( Insulin And Non Insulin Category ) In Life Satisfaction in Job Dimension

\begin{tabular}{|l|c|c|c|c|c|}
\hline Life Satisfaction & Df & Mean & SD & t-value & Sig. \\
\hline Job & 39 & 24.28 & 9.35 & 15.57 & $.01^{\text {** }}$ \\
\hline
\end{tabular}

Level of sig. $.01^{\star *}, .05^{\star}$

3. On social dimension of life satisfaction the t value is 25.19 (Table 5) again significant at higher level i.e. .01 on the basis of insulin non insulin category. In this dimension mean for diabetic men with insulin was higher (25.5) as compared to diabetic men non insulin category (24.45) showing that diabetic men with insulin had better life satisfaction on social dimension as compared to diabetic male non insulin category.

Table 5: T test

Differences between Diabetic Male ( Insulin and Non Insulin Category) In Life Satisfaction In Social Dimension

Level of Sig. $.01^{* *}, .05^{*}$

\begin{tabular}{|l|c|c|c|c|c|}
\hline Life Satisfaction & Df & Mean & SD & t-value & Sig. \\
\hline Social & 39 & 27.75 & 9.35 & 25.19 & $.01^{* *}$ \\
\hline
\end{tabular}

(4) The t value on marital dimension of life satisfaction among diabetic males insulin and non insulin category was found 20.43 significant at higher level (Table 6), On this dimension again diabetic men of non insulin category had higher mean of 27.65 as compared to diabetic male of insulin category (27) showing that diabetic male of non insulin category were higher on the marital dimension of life satisfaction. 
Table 6: T Test

Differences between Diabetic Male (Insulin And Non Insulin Category) In Life Satisfaction In Marital Dimension

Level of Sig. .01**, $.05^{\star}$

\begin{tabular}{|l|c|c|c|c|c|}
\hline Life Satisfaction & Df & Mean & SD & T-Value & Sig. \\
\hline Marital & 39 & 28.35 & 8.55 & 20.43 & $.01^{\star \star}$ \\
\hline
\end{tabular}

(5) The t value on family dimension of life satisfaction among diabetic males insulin and non insulin category 25.83 significant at higher level (Table 7), On this dimension diabetic men of insulin category had higher mean of 16.6 as compared to diabetic male of non insulin category 15.85 showing that diabetic male of non insulin category were higher on the marital dimension of life satisfaction.

Table 7: T test

Differences between Diabetic Male ( Insulin And Non Insulin Category) In Life Satisfaction In Family Dimension

\begin{tabular}{|l|c|c|c|c|c|}
\hline Life Satisfaction & Df & Mean & SD & t-value & Sig. \\
\hline Family & 39 & 16.64 & 8.55 & 25.83 & $.01^{* *}$ \\
\hline
\end{tabular}

\section{Discussion}

This study aimed at studying Life Satisfaction among different dimensions in the male patients of Diabetes (Insulin Vs non insulin) and studying the Happiness level among male patients of Diabetes ; Insulin Vs Non Insulin, results showed that there are significant differences among different dimensions of life satisfaction and happiness among male patients of diabetes of insulin Vs non insulin category, looking at the scores obtained in both scales. These outcomes are corroborated by previous studies by Humboldt, Leal, Santos \& Niculescu (2013), on Aging with Diabetes: Sense of Coherence and Satisfaction with Life in European Older Adults with Type 2 Diabetes that underline that presence of diabetes influences satisfaction with life(SWL) and sense of coherence (SOC), only diabetes' group had lowest SOC and SWL scores, this group also showed an association between SOC and SWL scores. In the 'without diabetes' and 'with diabetes and other' groups no correlation was found between SOC and SWL scores, In a similar study by Rubin and Peyrot (1999), it has been found that better glycemic control is related with better quality of life, no matter how intensive treatment is given, any kind of complication in diabetes had a detrimental effect on quality of life, similarly when patient's health status is improved and their perceived ability to control their disease was increased it showed improved quality of life in them.

In an other study by Shahin, kalawaet.al (2013) also reported significance difference in many items related to quality of life \&diabetic foot patients satisfaction between Egyptian \& Sudanese patients, Sudanese diabetic foot patients had low quality of life and satisfaction relate to their disease, supporting our study.

Thus, our findings that there are significant differences among different dimensions of life satisfaction and happiness among male patients of diabetes of insulin Vs non insulin category, looking at the scores obtained in both scales ,came as no surprise. 


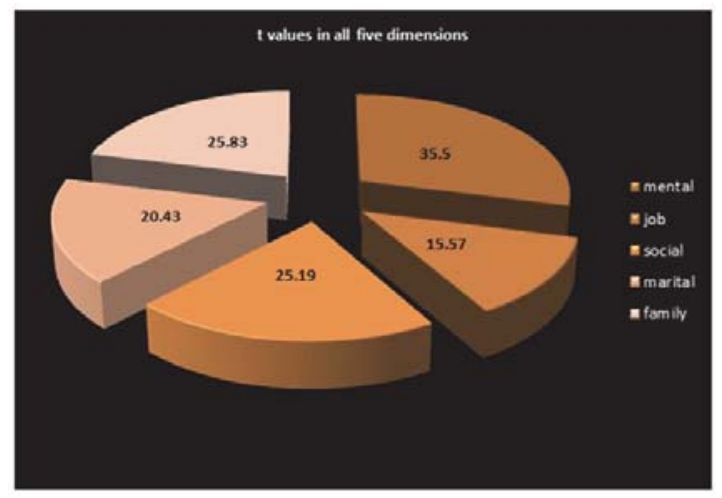

Figure 1: A Pie Diagram t values on all five dimensions namely (Mental, Job , Social ,Marital and Family )

\section{Conclusions}

The hypothesis of the paper was built on the premise that insulin being vigorous diabetic treatment should improve the life quality of the patient of which happiness and life satisfaction are the factors that could measured. Various dimension of life satisfaction should also bear impact of the treatment. With the analysis of data, following conclusions can be drawn -

1. On the contrary to hypothesis, happiness is found higher in diabetic males of non insulin category as compared to diabetic males of insulin category. This is one factor which is to analyzed further with larger samples, as there could be some interfering psychological factors such as, patient with insulin could be of opinion that they are more sick than noninsulin patients despite the fact that both could have same physiological conditions.

2. Diabetic male of insulin category were found higher in mental, social and marital dimensions while diabetic male of non insulin category were found higher on job and marital dimension of life satisfaction thus confirming the hypothesis.

\section{Contribution of the Paper}

This paper is only a step towards studying the impact of insulin on life standard of diabetic patients. The sample size however is small, with larger sample across various region of the country, including age groups, genders, their life style etc, the study may prove interesting and may lead to results which could help in deciding line of action while treating such patients.

\section{Suggestions and Recommendations}

(1) The diabetes happens to be a disease which requires lot of psychological support, while maintaining it. The study may further be enhanced with inclusion of male and female of various age groups and back grounds.

(2) It is of utmost importance to maintain quality of life of diabetic patient by controlling their glucose level with or without execution of insulin. Thus further study may include various behavior patterns such as anger, depression, escapism etc. to decipher the relations of any particular behavior to the level of glucose

(3) Study may be conducted taking the sample of insulin dependent patients who have adopted other method of controlling diabetes such as Yoga, mediations etc. This will help in maintaining better protocol or medication of the diabetic patients

(4) Maintaining quality of life of an diabetic patient being the ultimate goal of any therapy, study may further be enhanced to assess impact of insulin on happiness and life satisfaction with Cognitive Behavior Therapy, particularly where the behavior of a diabetic patient is not recognized as outcome of clinical situation due to low diabetic literacy.

(5) Reasons for happiness were not within the purview of the study but the suggestion made in review could be 
interesting as it will widen the study with other elements such as, diabetic literacy, financial status, food culture, social structure etc.

\section{Acknowledgements}

I sincerely acknowledge Dr Sachin Gupta Diabatologist at Bhopal for helping in data collection, heartfelt thanks to Vishwa Chandra Dubey for making this study possible.

\section{References}

Buetell, N. (2006). Life Satisfaction, a Sloan Work and Family Encyclopedia entry. Retrieved May 10, 2007, from the Sloan Work and Family Research Network website.

Cummins, R. A. (1997). Assessing Quality of Life for People with Disabilities. In R. Brown (Ed.), Quality of Life for people with disabilities: models research and practice. (2nd ed., pp. 116-150).

Diener E. (1984). Subjective well-being. Psychological Bulletin, 95(3), 542-575.

Diener, E., Emmons, R. A., Larsen, R. J., \& Griffin, S., (1985). The Satisfaction with Life Scale. Journal of Personality Assessment, 49, 71-157.

Erich Fromm (1947) Man for himself, an inquiry into the psychology of ethics Henry Holt and Company.

Erich Fromm (1990). The Sane Society Holt Paperbacks.

Farquhar, M. (1995). Definitions of Quality of Life: taxonomy, Journal of Advanced Nursing, 22(3), 502- 508.

Hughes, C., \& Hwang, B. (1996). Attempts to Conceptualize and Measure Quality of Life. (Vol.1). Washington: American Association on Mental Retardation

Muldoon, M. F., Barger, S. D., Flory, J. D., \& Manuck, S. B. (1998). What are Quality of Life Measurements Measuring? British Medical Journal, 316(7130), 542-545.

Macarena Vallejo Martín, Maria del Pilar Moreno-Jiménez (2012).An Evaluation of Life Satisfaction within the Migratory Experience According to Psychosocial Variables, Psychology 2012. Vol.3, No.12A, 1248-1253 Published Online December 2012 in Sci Res (http://www.SciRP.org/journal/psych) .

Martín, M. \& Moreno- Jiménez, M. (2012). An Evaluation of Life Satisfaction within the Migratory Experience According to Psychosocial Variables. Psychology, 3, 1248-1253.

Neugarten, B. L., Havighurst, R. J., \& Tobin, S. S. (1961). The Measurement of Life Satisfaction. Journal of Gerontology, 16, 134-143.

Richard R. Rubin and Mark Peyrot ( 1999); Quality of life and diabetes ; Diabetes/Metabolism Research and Reviews, Volume 15, Issue 3 pages 205-218

Seligman, Martin E.P. (1991). Learned optimism: how to change your mind and your life New York, NY: Pocket Books.

Seligman, Martin E.P. (1996). The Optimistic Child: proven program to safeguard children from depression \& build lifelong resilience. New York, NY: Houghton Mifflin.

Seligman, Martin E.P. (2002). Authentic Happiness: using the new positive psychology to realize your potential for lasting fulfillment New York, NY: Free Press.

Seligman, Martin E.P. (2004). "Can happiness be taught?” Daedalus, Spring 2004.

Seligman, Martin E.P. Doing the Right Thing: Measuring Well Being for Public Policy. International Journal of Wellbeing Vol. 1, No. (2011)

Seligman, M.E.P. (2004). Can Happiness be Taught?. Daedalus journal, Spring 2004. Escape from Freedom (U.S.), The Fear of Freedom (UK) (1941) ISBN 978-0-8050-3149-2.

Sofia von Humboldt, Isabel Leal , Susana Santos \& Georgeta Niculescu (2013) Aging with Diabetes: Sense of Coherence and Satisfaction with Life in European Older Adults with Type 2 Diabetes; Review of European Studies; Vol. 5, No. 1; 2013 ISSN 1918-7173 E-ISSN 1918-7181, URL: http://dx.doi.org/10.5539/res.v5n1p1

Suh, E.Diener, E., \& Fujita, F. (1996). Events and Subjective Well-being: only recent events matter. Journal of Personality and Social Psychology, 70(5), 1091-1102.

Akahashi M,Araki A, Watanabe S, Haga H, Kimbara Y, Tamura Y, Chiba Y, Mori S, Ito H,Shibata H(2010); Community Participation is Associated with Life Satisfaction in Elderly People with Diabetes Mellitus. Nihon Ronen Igakkai Zasshi.Japanese Journal of Geriatrics , 47(2):140-146

Veenhoven. R. (1984) Conditions of Happiness, Dordrecht: Reidel, (reprinted 1991 by Kluwer Academic.)

Veenhoven.R. (1996). Developments in Satisfaction Research. Social Indicators Research, 37, 1-46

http://en.wikipedia.org/wiki/Happiness

http://www.pursuit-of-happiness.org/history-of-happiness/martin-seligman-positive-psychology/

https://scholarworks.iupui.edu/bitstream/handle/1805/1160/Barrett_\%26_Murk_Life\%20Satisfaction.pdf?sequence=1 